\title{
Erratum: Stellar properties and nuclear matter constraints [Phys. Rev. C 93, 025806 (2016)]
}

\author{
Mariana Dutra, Odilon Lourenço, and Débora P. Menezes \\ (Received 22 September 2016; published 21 October 2016)
}

\section{DOI: 10.1103/PhysRevC.94.049901}

In this Erratum we show the corrected results related to two density-dependent relativistic mean-field parametrizations analyzed in a previous work and reexamine some of our conclusions, mainly the one that three models produce stars with maximum masses above the recent observational results, whereas in fact, only two of them can do so. In the original paper, we analyzed the stellar

TABLE I. The corrected results of DDH $\delta$ and DD-ME $\delta$ for neutron star main properties: maximum stellar masses in terms of $M_{\odot}\left(M_{\max } / M_{\odot}\right)$, the radius of the corresponding star $(R)$, the radius of a star with a $1.44-M_{\odot}$ mass $\left(R_{1.44} M_{\odot}\right)$, and the central energy density of the maximum mass star $\left(\varepsilon_{c}\right)$. Properties related to the direct Urca (DU) process: mass of the star in terms of $M_{\odot}$ at the onset of the DU process $\left(M_{\mathrm{DU}} / M_{\odot}\right)$, related baryonic density $\left(\rho_{\mathrm{DU}}\right)$, and proton fraction $\left(Y_{\mathrm{DU}}\right)$.

\begin{tabular}{lcccccccc}
\hline \hline Model & Reference & $M_{\max } / M_{\odot}$ & $R(\mathrm{~km})$ & $R_{1.44 M_{\odot}}(\mathrm{km})$ & $\varepsilon_{c}\left(\mathrm{fm}^{-4}\right)$ & $M_{\mathrm{DU}} / M_{\odot}$ & $\rho_{\mathrm{DU}}\left(\mathrm{fm}{ }^{-3}\right)$ & $Y_{\mathrm{DU}}$ \\
\hline & & & $\vdots$ & & & \\
DDH $\delta$ & {$[3]$} & 2.142 & 11.138 & 12.587 & 6.412 & 1.876 & 0.569 \\
DD-ME $\delta$ & {$[4]$} & 1.958 & 10.241 & 11.836 & 7.714 & 1.793 & 0.765 & 0.138 \\
\hline \hline
\end{tabular}

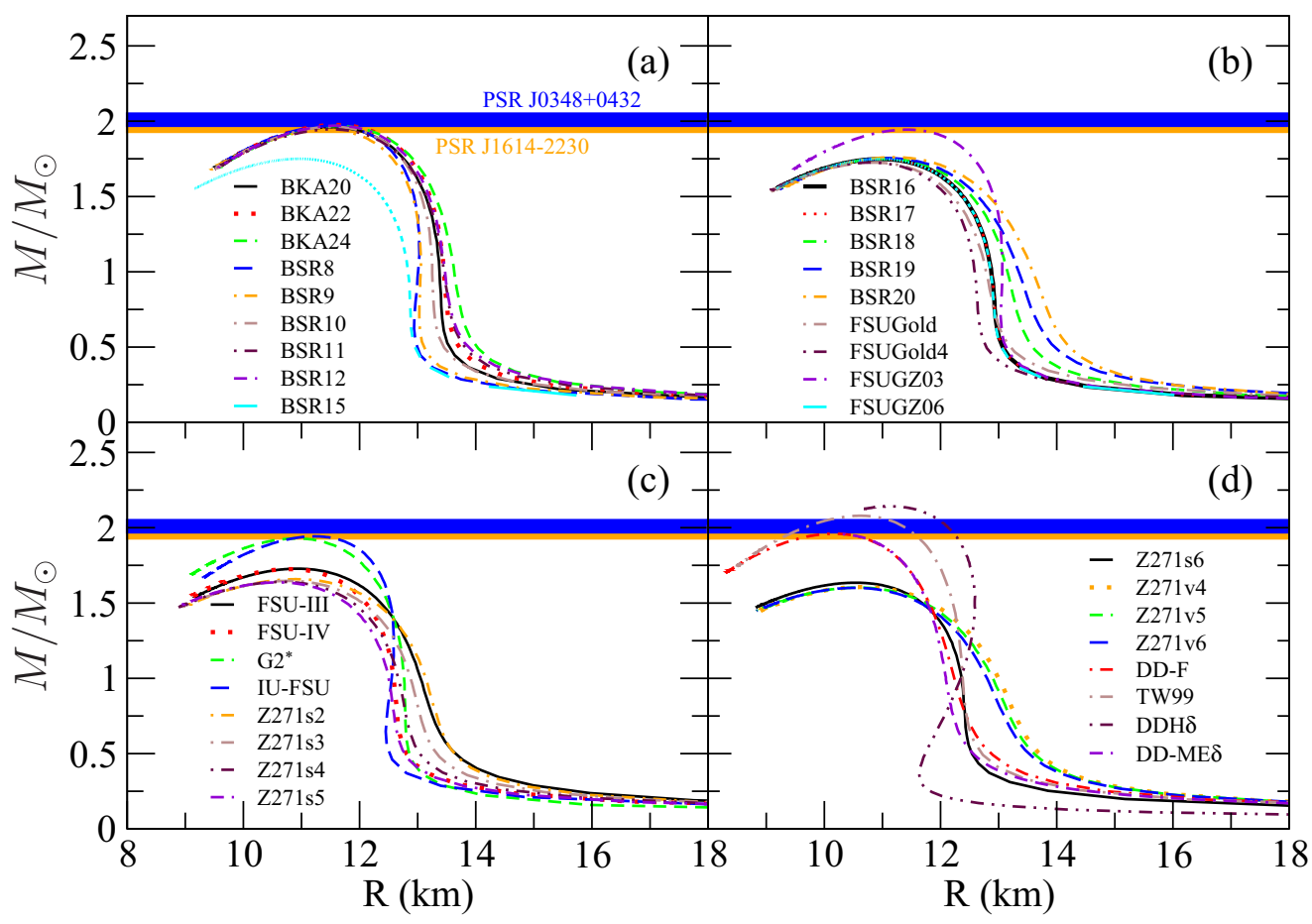

FIG. 1. Neutron star mass-radius relation. The horizontal bands indicate the masses of PSR J1614-2230 [1] (orange line) and PSR J038 + 0432 [2] (blue line).

TABLE II. Main properties of a neutron star with hyperonic matter.

\begin{tabular}{lcccr}
\hline \hline Couplings & Model & $M_{\max } / M_{\odot}$ & $R(\mathrm{~km})$ & $R_{1.44 M_{\odot}(\mathrm{km})}$ \\
\hline SET 1 & TW99 & 1.895 & 9.631 & 11.015 \\
SET 2 & TW99 & 1.700 & 10.166 & 11.432 \\
SET 1 & DDH $\delta$ & 1.859 & 10.039 & 11.790 \\
SET 2 & DDH $\delta$ & 1.689 & 10.580 & 12.456 \\
\hline \hline
\end{tabular}




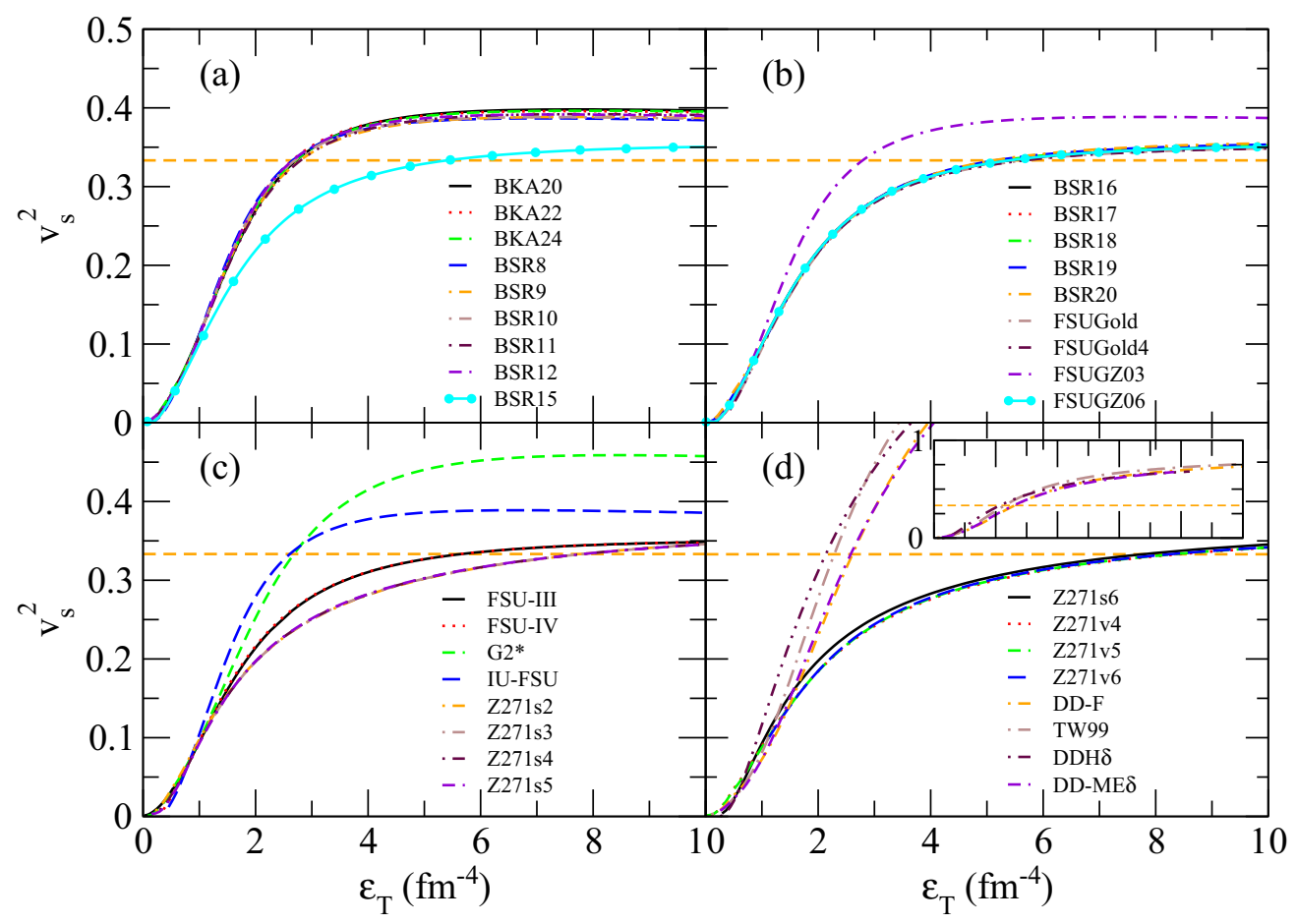

FIG. 2. Squared sound velocity for nuclear matter in the $\beta$ equilibrium. The orange dashed line represents the limit $v_{s}^{2}=\frac{1}{3}$.

properties of the relativistic mean-field parametrizations shown to be consistent with nuclear matter, pure neutron matter, and symmetry energy constraints. Among our conclusions, we stated that three models yield neutron stars with maximum masses above that pointed out by the recent observational results of about $2 M_{\odot}$ [1,2]. Unfortunately, there was a technical mistake in the calculation of the maximum stellar masses for two of the parametrizations with density-dependent couplings and $\delta$ mesons, namely, DDH $\delta$ and DD-ME $\delta$, which resulted in the wrong conclusions. The corrected tables and figures are presented here. In corrected Table I, we show only the entries related to these two parametrizations.

Note that the corrected values for the maximum stellar masses modified from 12 to 13 the number of parametrizations that produce neutron stars with maximum mass in the range of $1.93 \leqslant M / M_{\odot} \leqslant 2.05$. They are as follows: BKA20, BKA22, BKA24, BSR8, BSR9, BSR10, BSR11, BSR12, FSUGZ03, G2*, IU-FSU, DD-F, and DD-ME $\delta$. Now, only two parametrizations, TW99 and DDH $\delta$, yield maximum masses above the bands shown in Fig. 1.

Another correction was made in the results of Sec. II C. Now, the inclusion of hyperons in the DDH $\delta$ parametrization generates, for both sets (SET1 and SET2), maximum masses lower than the range of $1.93 \leqslant M / M_{\odot} \leqslant 2.05$, see Table II. Note also the same behavior presented by DDH $\delta$ and TW99 parametrizations, namely, the larger reduction in the maximum neutron star mass verified when SET2 is considered.

For the sake of completeness, we have also reanalyzed the sound velocity. The corrected curves for the DDH $\delta$ and DD-ME $\delta$ parametrizations are depicted in Fig. 2 as follows. The conclusions drawn in the original paper remain basically the same in this case.

This work was partially supported by CNPq under Grant No. 300602/2009-0. O.L. acknowledges support from CNPq. M.D. acknowledges support from Fundação de Amparo à Pesquisa do Estado do Rio de Janeiro (FAPERJ), Grant No. 111.659/2014. We would like to thank Prof. C. Providência from the University of Coimbra, Portugal, for drawing our attention to the mistake in our calculations.

[1] P. B. Demorest, T. Pennucci, S. M. Ransom, M. S. E. Roberts, and J. W. T. Hessels, Nature (London) 467, 1081 (2010).

[2] J. Antoniadis, P. C. C. Freire, N. Wex et al., Science 340, 448 (2013).

[3] T. Gaitanos, M. Di Toro, S. Typel, V. Baran, C. Fuchs, V. Greco, and H. H. Wolter, Nucl. Phys. A 732, 24 (2004).

[4] X. Roca-Maza, X. Viñas, M. Centelles, P. Ring, and P. Schuck, Phys. Rev. C 84, 054309 (2011). 